\title{
Video Rasterstereography of the Spine and Pelvis in Eight Erect Positions: A Reliability
}

\section{Study}

\section{Abstract}

\section{Introduction}

To investigate the reliability and variability of Video Rasterstereography (VR) measurements of the spine and pelvis, for eight proposed standing postures, in order to help define an optimal standing position for erect pelvis radiography.

\section{Methods}

Surface topography data were collected using the formetic 4D dynamic modelling (Diers) system. 61 healthy participants were recruited; each participant performed eight different standing positions. Four positions were performed with the feet shoulder width apart and parallel, and four positions were performed with the feet shoulder width apart and internally rotated. For the upper extremity, each of the (two sets of) four positions were performed with different arm positions (arms by the sides, arms crossed over the chest, arms $30^{\circ}$ flexed and touching the medial end of the clavicle, arms $30^{\circ}$ flexed with the hands holding a support). Three sets of surface topography were collected in the eight positions $(n=24)$. The variability was assessed by calculating standard error of the measurement (SEm) and the coefficient of variation (CV). Reliability was assessed using intra-class correlation coefficients $(\mathrm{ICC} \pm 95 \% \mathrm{CI})$.

\section{Results}

No significant differences in the SEm were found between the three paired measurements for all standing positions $(P>0.05)$. ICC values demonstrated excellent reliability for all measurements across the eight standing positions (range 0.879 to 1.00 [95\% $\mathrm{Cl} 0.813-1.00]$ ).

\section{Conclusion}

Evaluating eight standing positions radiographically would be unethical as it would involve repeat radiation exposures. Using the formetic 4D dynamic modelling (Diers) system, provides an alternative and has shown that there was only a minimal, non-statistically significant, differences between the eight different standing positions.

Key words: Video Rasterstereography, pelvis tilt, erect pelvis radiograph, posture. 


\section{Introduction}

For spine and pelvis disorders, imaging by projection radiography is still the standard method for diagnosis, monitoring and follow up ${ }^{1}$. An anteroposterior (AP) pelvis X-ray image is commonly undertaken with the patient in the supine position for the investigation of hip pathologies. As hip pain often presents during weight bearing and daily functional activities, such as walking and running, several studies argue that the imaging of the pelvis must be achieved in a standing position, in order to provide more clinically useful information ${ }^{2,3}$. Previous studies have reported that there are changes in the pelvis measurements as the posture changes i.e. moving from supine to standing ${ }^{4-8}$. Moreover, there is no currently accepted 'standard protocol' for erect pelvis radiography and it is not clear whether different standing positions commonly used to obtain standing X-ray images of the pelvis could be associated with differences in postural alignment ${ }^{9}$. It is also important to understand the effects of different standing positions on the spine when acquiring pelvis $X$-ray images. With the pelvis articulating with the lumbar spine at the sacroiliac joint, standing positions could affect spine measurements, which in turn may influence pelvic metrics. It is important to understand such postural variations as these could help when obtaining $\mathrm{X}$-ray images in the optimal position. Such variations may influence the diagnosis and treatment outcomes for patients with pelvis disorders, such as pincer impingement and osteoarthritis (OA). For instance, pelvic tilt (PT) is defined by the angle between the line connecting the anterior superior iliac spine (ASIS) and the posterior superior iliac spine (PSIS), and a horizontal line ${ }^{10}$. As anterior PT increase the lumbar lordosis (LL) increases ${ }^{11}$ which increases the load on the lumbar spine ${ }^{12}$. Acetabular retroversion is one of the early signs of hip OA and it varies with PT. PT influences the presence or absence of a retroversion sign 13 which can also affect diagnosis. Correct positioning, therefore, has implications in the diagnosis, monitoring and follow-up of patients with hip conditions.

For a patient who has to undergo total hip replacement (THR), the increased PT (rotation around the transverse axis) results in significant decrease in cup anteversion and vice versa ${ }^{14}$. These variations have a significant effect on the precision of the acetabular cup position - which can lead to instability, wear and osteolysis ${ }^{15}$. Moreover, even in normal people without any abnormality, if pelvic X-ray images are obtained with excessive PT then this can lead to false diagnosis ${ }^{16}$. This has many disadvantages such as influencing the correct diagnosis of femoroacetabular impingement (FAI), and it can also affect the recommendation for surgical treatment. Clinically, patients who underwent THR are traditionally evaluated by supine imaging. However, 
as they move into an erect position their pelvis tilts backwards, therefore, cup inclination and anteversion can become markedly deviated from the safe zone ${ }^{17,18}$

Over the past three decades, there have been many devices developed for spinal and pelvis postural assessments. The main advantages of some of these methods are that they are non-invasive and free from the use of ionising radiation, for example photometric imaging, mechanical inclinometry and video rasterstereography (VR) back reconstruction devices. The latter are considered particularly advantageous since they require a minimal role of the operator since they do not require markers and complex detectors. Such systems were originally developed by Drerup and Hierholzer in the $1980 \mathrm{~s}^{19}, 20$. The basic principles of these devices depend on triangulation, consisting of two cameras that 'view' the patient from two different angles. A projector shines parallel white lines on the surface of the back. The camera detects the deformity of the parallel lines that occur as a result of the concave and convex shape of the back. Using such data, it is possible to quantitatively map the morphology of the spine and pelvis and obtain a series of automated measurements ${ }^{21}$. These measurements can serve as valuable quantitative data when assessing changes to the anatomical alignment of pelvis during different standing positions. Previous such VR systems have not been used to evaluate the suitability of different erect positions that could be used during radiographic examinations.

The aim of this study was to evaluate the reliability and variability of VR measurements of the pelvis and spine in different standing positions in order to help inform the technique for AP erect pelvis radiography. Using such data, a comparison of morphological measurements between standing positions could help identify an optimum position for erect pelvis radiography which is currently not present in the literature. The positions that have been used within this study were identified from previous research studies which sought to evaluate spinal parameters but not specifically pelvis parameters ${ }^{22,23}$. Additionally, these positions were considered to represent a range of common standing positions that could be used during erect pelvic X-ray imaging. Internal rotation of the feet is traditionally recommended when performing pelvis radiography in order to provide more information about femoral head and neck ${ }^{24}$ and was included as a variable within this research. Supports are often provided for patients with balance and mobility issues and as such should be included within any evaluation of standing positions. 


\section{Methods}

\section{Subjects}

Ethical approval to conduct this study was granted from the University of Salford (HSR1617-142). Healthy volunteers were recruited by email and paper flyer. Adult ( $\geq 18$ years) volunteers with a healthy skeletal system, not having been injured (in the past six months), free from previous spinal or pelvic surgery, known neurological problems or discrepancies in leg length, were included. Participants were excluded if they had a history of pelvic or spinal abnormalities, fractures, history of serious trauma or previous pelvic surgery. Participants with known secondary degenerative changes in the spine and hip, THR or significant low back / pelvic pain were also excluded.

All participants were informed of the aim and requirements of the study, and all signed a consent form. Demographic characteristics of the participants are presented in Table 1. A total of 61 healthy volunteers ( 27 men, 34 women) participated in this study. Data were anonymised after the Diers acquisitions and then analysed.

A repeated measures ANOVA was run using previously acquired pilot study data in the $G$ power computer programme. The alpha value set to 0.05 , power 0.85 and this indicated that 20 participants were needed to conduct this study. This study aimed to assess the impact of different body habitus on PT, and as the body weight increase the influence on the pelvis and lumbar spine could differ therefore, the sample size included three different groups according to BMI. Underweight (BMI<18.5) and normal weight (BMI 18.5 - 24.99) were one group, overweight $\mathrm{BMI} \geq 25$ and obese $\mathrm{BMI} \geq 30$ were groups two and three. With the power calculation originally indicating the need for 20 participants this was increased to 60 to represent the three BMI groups.

\section{Equipment}

Surface topography data was collected using the formatic 4D dynamic modelling system (Diers International $\mathrm{GmbH}$, Schlangenbad, Germany). The Diers system has a high resolution for back shape reconstruction (reconstruction error $0.2-0.5 \mathrm{~mm}$; resolution $10 \mathrm{pts} / \mathrm{cm} 2)^{25}$. The system makes the measurements depending on specific back and pelvis landmarks, including the $7^{\text {th }}$ cervical vertebral prominence (VP), left and right pelvis dimples (DR, DL) which represents the posterior superior iliac spines (PSIS) and the sacrum point (SP). The basis of this system depends 
on triangulation equations that produce three-dimensional (3D) surface reconstructions. The device acquires the images by projecting lines of white light on the back and then a digital image of this is created by the computer. The Diers system measures patients over a six-second interval, taking two images per second. The 12 images acquired are evaluated and averaged by the machine's software, correcting for any subject movement during the data acquisition period $^{26}$. A sample of 3D images of the spine and pelvis are shown in 
Figure 1. The precision of the mathematical function behind this device has been further enhanced to reflect the correct 3D spinal shape by using more sophisticated equations ${ }^{27,} 28$. Error! Reference source not found. illustrates the Diers measurements of the back and pelvis.

\section{Protocol}

Before commencing data collection, the consistency in positioning both between and within the subjects was considered. A bespoke foot plate was constructed to help decrease the variation between and within the subjects. An adjustable 'foot print' assured the reproducibility of the standing position by standardising shoulder width and providing $20^{\circ}$ internal rotation of the feet. For standing positions, which involved the use of a hand support, the level of the hand position was marked with tape, so all subjects held onto the same place and at the same angle. The floor was marked with a permanent marker, so that the position of the foot plate and hand support was constant for the duration of the study.

Before starting data collection participants were asked to change clothes in a private changing room, this included removal of the upper garments and wearing a special gown (revealing a bare posterior surface). Each participant also changed their lower clothing and wore sport shorts which facilitated the visualisation and palpation of PSIS. Reflective markers were positioned on the right and left PSIS and on the $7^{\text {th }}$ cervical VP by a study researcher. Markers were used instead of the automatic detection of bony landmarks to aid reproducibility and was recommended by the manufacturer, and has been used in a previous study ${ }^{26}$. Height, weight and BMI were recorded before starting the Diers measurements. Following this, each participant was placed in eight different standing positions which were demonstrated by the researcher to ensure participant compliance and reproducibility

\section{Table 2.}

Participants were asked to stand in a relaxed state with their head facing forward. After completing the Diers acquisition for one position, the participants were asked to walk around to give them an opportunity to relax and better simulate the participant's normal posture ${ }^{29}$. Measurements were initial performed in each of the different standing positions (1 to 8 ). Between each position participants were allowed to have a short break and move around the room. Once all eight positions were evaluated this process was then repeated on two further occasions, during the same session. This gave a total of 24 measures for each pelvic / spinal 
parameter. Following image acquisition, the Diers system automatically generated different measurements of the spine and pelvis such as: pelvic tilt (PT), pelvic torsion (PTor), thoracic kyphosis (TK), lumbar lordosis (LL) and dimple distance (DD). To understand the reliability of the Diers system it is necessary to define the parameters measured by the device: -

- Thoracic Kyphotic angle $\left({ }^{\circ}\right)($ TK) - maximum thoracic angle calculated from ICT (inflectional point of the curvature from cervical to thoracic spine) and ITL (inflectional point of the curvature from thoracic to lumbar spine).

- Lumbar Lordosis angle $\left({ }^{\circ}\right)(\mathrm{LL})$ - is the maximum lumbar angle calculated from ITL and ILS (inflectional point of the curvature from lumbar to sacral spine)

- Pelvic tilt angle $\left({ }^{\circ}\right)(\mathrm{PT})$ - is the angle between a vertical plumb line and the tangent on the lumbar dimples (DL and DR) in the frontal plane.

- Pelvic torsion angle $\left({ }^{\circ}\right)$ (PTor) - is the torsion between left and right side of the pelvis bones.

- Dimple distance (mm) (DD) - is the distance between the two pelvic dimples (DL and DR). The measurements selected above are the most common parameters measured within the literature ${ }^{26,30-32}$ and would provide an indication of the three-dimensional (3D) orientation of the pelvis in different standard positions. Moreover, it has been reported in previous work that using the standard deviation of intra-individual lumbar dimple distance was an accurate parameter for evaluating positional variation ${ }^{33}$.

A 3D image was reviewed after each acquisition; a repeat was done if required. The validity of the Diers system has been previous established in a number of clinical studies ${ }^{27,29,30,32,34-36}$.

\section{Statistical Analysis}

All data were inputted into an Excel spreadsheet (MS Excel 2016, Microsoft Corp, Redmond, WA). Normality was checked visually and using the Shapiro-Wilk test. Normally distributed data were presented as mean values plus or minus their respective standard deviations (SD). Data with a non-parametric distribution were represented by median values together with their interquartile ranges (IQR). Differences between the group means were examined using either a oneway analysis of variance (ANOVA) for repeated measures or the Friedman test. For reliability, the Intra-Class-Correlation coefficient, with $95 \%$ confidence intervals (ICC $\pm 95 \% \mathrm{Cl}$ ) was used to compare the three repeated measures for each position. An ICC more than 0.90 indicates high reliability, 0.80-0.89 indicates good reliability, 0.70-0.79 fair and poor reliability is less than $0.69^{30}$. For variability, the standard error of measurement (SEm) and the coefficient of variation 
(CV) were also reported. Statistical calculations were performed using the statistical software package SPSS v. 22.0 (IBM Inc, Armonk, NY). Statistical significance was determined as $p<0.05$.

\section{Results}

The mean (average of three repeated measurements in one day) DD, PT, PTor, TK and LL values and their respective standard deviations are presented in Table 3 for all participants in the eight different standing positions. The stability of the Diers system measurements were evaluated by calculating the group mean differences and comparing them inferentially using a repeated measures ANOVA. The results indicated that there were no significant changes within the group means between the three paired positions, for both spine and pelvis measures ( $P>0.05$; Table 3 ).

\section{Variability}

Intra-subject variability of the three repeated measures demonstrated only very small variations in the SEm (range 0.00 to 0.31 ; Table 33 ). The largest SEm was for the kyphosis angle $\left(0.31^{\circ}\right)$ while the smallest was the dimple distance $(0.00 \mathrm{~mm})$. Combining the $\mathrm{SEm}$ and the $\mathrm{CV}$ values indicated that the dimple distance had the smallest measurement variability between all five measures and for all positions (SEm $0.00 \mathrm{~mm}, \mathrm{CV} 0 \%$ ). For PT, the SEm and CV ranged from 0.05 to $0.19 \mathrm{~mm}$ and $1 \%$ to $10 \%$, respectively which was the greatest variation amongst the five different measurements. Kyphosis angle had the smallest CV (2\% to $4 \%$ ). The SEm for lordosis angle ranged from $0.08^{\circ}$ to $0.24^{\circ}$ (CV $2 \%$ to $8 \%$ ). Pelvis torsion had a CV from $1 \%$ to $6 \%$ and a SEm from $0.16^{\circ}$ to $0.30^{\circ}$.

\section{Reliability}

The reliability for the assessment of the five spinal and pelvic parameters was excellent with ICCS ranging from 0.88 to $1.00(95 \% \mathrm{Cl} 0.81$ to 1.00$)$ for all standing positions 
Table . ICC values for dimple distance ranged from 0.97 to 1.00 for all eight positions. ICC for pelvis torsion ranged from 0.88 to 0.96 and for pelvis tilt was 0.94 to 0.99 . ICC values for the kyphosis and lordosis angles, were similar, and ranged from 0.97 to 1.00 and 0.98 to 1.00 , respectively. 


\section{Discussion}

The reliability of the Diers system for spinal and pelvis measurements has been previously described in the literature $27,29,30,32,34-36$. In our study, the aim was to evaluate its reliability in assessing measurements in eight different standing positions to help identify an optimal standing position for pelvis standing radiography. Video rasterstereography is a relatively cheap, fast, non-invasive and radiation free measurement method that has been used extensively to evaluate spine and pelvis posture ${ }^{36}$. The results from this study indicate that for eight different standing positions, the Diers shows high reliability. Therefore, when based on reliability data each of the suggested eight standing positions could be used for standing pelvis radiography without affecting spine and pelvis morphology. Before any definitive recommendations can be made it is important to evaluate the radiographic imaging appearances and radiation dose implications of the different standing positions alongside the VR data. It is also important to note that our VR data were acquired using healthy volunteers and further clinical validation is required.

According to the ANOVA results, which evaluated the mean measurement differences between paired measures $(n=3)$ for the same position, no statistically significant differences were found for any of the five spine and pelvis parameters and across the eight different standing positions. To the authors' knowledge there have been no previous studies focusing on the reliability of VR for multiple standing positions. The majority of positions examined in this study have not been previously evaluated with the Diers system, with two exceptions where the feet are parallel and the upper extremities by the sides (position one), which considered to be the optimal posture for the Diers system, and the feet are parallel and the arms on the fits (position three). As a result, there is very limited comparable research published within the literature, however, comparison of position \#1 is, however, possible. The SEm, for position \#1, in our study was smaller than that obtained by Schroeder ${ }^{30}$ for PT, PTor, TK and $\operatorname{LL}\left(0.7 \mathrm{~mm}, 0.4^{\circ}, 0.9^{\circ}\right.$ and $0.8^{\circ}$, respectively). A possible explanation for this is the control of variation within and between participants among the positions; as noted earlier this was achieved through use of the footplate and hand support. It must be noted that the work by Schroeder ${ }^{22}$ did not include the use of reflective markers on the skin surface and this could also affect the comparability of our results. Moreover, Schroeder et al. investigated instantaneous repeated assessments and those within two days, and furthermore within one-week distance, which explains a greater variability affecting SEM 
and $\mathrm{CV} \%$ and also the ICCs. However, previous research done by Knott et.al. comparing between the using reflective marker and letting the Diers automatically detect the anatomical land marks, the results shows there is no significant differences in 12 parameters before using the reflective markers ${ }^{37}$. Moreover, all the reflective markers placed in this study were by one researcher who has more than 10 years' experience as a radiographer, these landmarks are frequently used in radiography. The CV in our study was greater than that of Schroeder, except for PTor (6\% versus 48\%). Furthermore, within our study SEm did not exceed $0.5 \mathrm{~mm} / 0.5^{\circ}$ for all measurements (maximum TK $0.23^{\circ}$ and minimum LL $0.09^{\circ}$ ).

In the study undertaken by Tabard-Fouge ${ }^{\prime} \mathrm{re}^{32}$ the researchers used reflective markers as in our study with the position for the participants replicating position number three in our study. They also undertook a number of comparable measurements including LL, TK and PT. Their results were similar to our results demonstrating high reliability in LL and TK (ICC 0.86 and TK 0.94 respectively). While for the PT their results show acceptable reliability (ICC 0.5 ). This was in slight contrast to the PT data generated in our study which showed high reliability, this could have resulted from using the bespoke foot plate which helped in ensuring consistency when positioning each participant.

Previous studies that have investigated the reliability of VR did not consider within participant variability ${ }^{29}$ and did not report CV values ${ }^{35}$. The SEm was evaluated using a spinal mouse (inclinometer) in a study to assess within participant reliability ${ }^{38}$. The SEm was higher in this study, 4.2 and 2.5 for TK and LL respectively, when compared to lower values encountered in our study $\left(\mathrm{TK}=0.23^{\circ}, \mathrm{LL}=0.09^{\circ}\right)$. Reasons behind the higher variability are not related to the system itself rather the effect of personal experience of the operator who performs the measurements together with the effect of spinal posture ${ }^{30}$.

When considering the ICC values, our results indicated similar reliability to those reported in the study by Schroeder ${ }^{30}$ who assessed the reliability of Diers system during the same day, between days and between weeks for PT, TK and LL which were 0.82, 0.98, 0.99, respectively. However, they reported lower levels (fair) reliability for PTor (ICC=0.78) whereas in our study this was higher at 0.96. Dimple Distance recorded the highest ICC values ranging from 0.99 to 1.00 for all positions. Previous reliability studies have not considered the dimple distance and comparisons are, therefore, problematic. Measurements of PT and PTor are more likely to be affected by overlying soft tissue and this 
would also be a factor for DD measurements ${ }^{30}$, our results showed high reliability for repeated measures, comparable with other published works ${ }^{30}$.

Within this study the volunteers were healthy and together with some variations in $\mathrm{BMI}$ and age would have some differences to the types of patients typically undergoing pelvic radiography. We believe that it is important to acknowledge this point but also that it was important to develop research on a healthy population first. Findings from our work are important in developing an evidence base for erect pelvic radiography but as stated further studies, involving patients with suspected or known pelvic/hip pathologies are warranted.

Patient variability is also an issue within our research and worthy of further discussion. Age related changes, $\mathrm{BMI}$ and co-existing pathologies are likely to affect the ability of a person to stand in a prescribed position. Whilst this work focused on healthy volunteers it should be expanded to those in the aforementioned groups in order to provide a comprehensive assessment of an erect pelvis radiographic position.

\section{Conclusion}

The Diers system, as a VR method, provided high reliability for the assessment of pelvis and spine measurements for different standing postures in healthy people. Data from this research is reliable and has provided evidence to support positional variations which could be used during standing radiography of the pelvis and spine.

This feasibility study suggests that each of the positions used within this study could be used for radiographic standing pelvis image acquisition without affecting spinal or pelvic anatomical alignment. However, the position with internal rotation of the feet and with the arms positioned in the support is a likely recommendation which would assist in visualisation of femur head / neck junction and also provide a stable standing position. Larger scale clinical studies are required to validate the impact of patient posture on the appearance of a range of anatomical structures, clinical outcome measures and the radiation dose from examination.

\section{References}

1 S. Langensiepen, O. Semler, R. Sobottke, et al., Measuring procedures to determine the Cobb angle in idiopathic scoliosis: A systematic review, Eur. Spine J. 22(11), 2360- 
2371 (2013).

A. Troelsen, L. Rømer, S. Jacobsen, S. Ladelund, and K. Søballe, Cranial acetabular retroversion is common in developmental dysplasia of the hip as assessed by the weight bearing position, Acta Orthop. 81(4), 436-441 (2010).

P.M. Courtney, C.M. Melnic, M. Howard, A. Makani, and N.P. Sheth, A Systematic Approach to Evaluating Knee Radiographs with a Focus on Osteoarthritis, J Orthop. ... 1(2), 1-6 (2014).

J. Pierrepont, G. Hawdon, B.P. Miles, et al., Variation in functional pelvic tilt in patients undergoing total hip arthroplasty, Bone Joint J. 99-B(2), 184-191 (2017). M. Khan, T. Beckingsale, M. Marsh, and J. Holland, Difference in the acetabular cup orientation in standing and supine radiographs, J. Orthop. 13(3), 168-170 (2016). T.J. Jackson, A.A. Estess, and G.J. Adamson, Supine and Standing AP Pelvis Radiographs in the Evaluation of Pincer Femoroacetabular Impingement, Clin. Orthop. Relat. Res. 474(7), 1692-1696 (2016).

A. Pytiak, J.D. Bomar, J.B. Peterson, et al., Analysis of spinal alignment and pelvic parameters on upright radiographs: implications for acetabular development, J. Hip Preserv. Surg. 3(3), 208-214 (2016).

S. Tamura, H. Miki, K. Tsuda, et al., Hip range of motion during daily activities in patients with posterior pelvic tilt from supine to standing position, J. Orthop. Res. 33(4), 542-547 (2015).

K. Alzyoud, P. Hogg, B. Snaith, K. Flintham, and A. England, Optimum Positioning for Anteroposterior Pelvis Radiography: A Literature Review, J. Med. Imaging Radiat. Sci. 49(3), 316-324.e3 (2018).

S. Sprigle, N. Flinn, M. Wootten, and S. McCorry, Development and testing of a pelvic goniometer designed to measure pelvic tilt and hip flexion, Clin. Biomech. 18(5), 462465 (2003).
D. Levine and M.W. Whittle, The Effects of Pelvic Movement on Lumbar Lordosis in the Standing Position, J. Orthop. Sport. Phys. Ther. 24(3), 130-135 (1996).

S.J. Preece, P. Willan, C.J. Nester, P. Graham-Smith, L. Herrington, and P. Bowker, 
Variation in Pelvic Morphology May Prevent the Identification of Anterior Pelvic Tilt, J. Man. Manip. Ther. 16(2), 113-117 (2008).

M. Tannast, S. Fritsch, G. Zheng, K.A. Siebenrock, and S.D. Steppacher, Which Radiographic Hip Parameters Do Not Have to Be Corrected for Pelvic Rotation and Tilt?, Clin. Orthop. Relat. Res. 473(4), 1255-1266 (2015).

T. Ala Eddine, H. Migaud, C. Chantelot, A. Cotten, C. Fontaine, and A. Duquennoy, Variations of pelvic anteversion in the lying and standing positions: Analysis of 24 control subjects and implications for CT measurement of position of a prosthetic cup, Surg. Radiol. Anat. 23(2), 105-110 (2001).

H. Yun, W.S. Murphy, D.M. Ward, G. Zheng, B. Hayden, and S.B. Murphy, Effect of pelvic tilt and rotation on cup orientation in standing anteroposterior radiographs, HIP Int. 112070001983166 (2019).

S. Anda, S. Svenningsen, T. Grontvedt, and P. Benum, Pelvic inclination and spatial orientation of the acetabulum, Acta radiol. 31(4), 389-394 (1990).

D. Bhaskar, A. Rajpura, and T. Board, Current Concepts in Acetabular Positioning in Total Hip Arthroplasty., Indian J. Orthop. 51(4), 386-396 (2017).

T. Scheerlinck, Cup positioning in total hip arthroplasty, Acta Orthop. Belg. 80, 336347 (2014).

B. Drerup and E. Hierholzer, Objective determination of anatomical landmarks on the body surface: Measurement of the vertebra prominens from surface curvature, J. Biomech. 18(6), (1985).

B. Drerup and E. Hierholzer, Automatic localization of anatomical landmarks on the back surface and construction of a body-fixed coordinate system, J. Biomech. 20(10), 961-70 (1987).

T. Huysmans, B. Haex, R. Van Audekercke, J. Vander Sloten, and G. Van Der Perre, Three-dimensional mathematical reconstruction of the spinal shape, based on active contours, J. Biomech. 37(11), 1793-1798 (2004).

M. Marks, C. Stanford, and P. Newton, Which lateral radiographic positioning technique provides the most reliable and functional representation of a patient's 
sagittal balance?, Spine (Phila. Pa. 1976). 34(9), 949-954 (2009).

F.D. Faro, M.C. Marks, J. Pawelek, and P.O. Newton, Evaluation of a functional position for lateral radiograph acquisition in adolescent idiopathic scoliosis, Spine (Phila. Pa. 1976). 29(20), 2284-2289 (2004).
A. Whitley, G. Jefferson, G. Hoadley, and C. Sloane, Clark's positioning in radiography, in 12th ed.(CRC Press, London: Arnold., 2005).

B. Drerup and E. Hierholzer, Back shape measurement using video rasterstereography and three-dimensional reconstruction of spinal shape, Clin. Biomech. 9(1), 28-36 (1994).

P. Knott, P. Sturm, B. Lonner, et al., Multicenter Comparison of 3D Spinal Measurements Using Surface Topography with Those from Conventional Radiography, Spine Deform. 4(2), 98-103 (2016).

J.W. He, Z.H. Yan, J. Liu, et al., Accuracy and repeatability of a new method for measuring scoliosis curvature, Spine (Phila. Pa. 1976). 34(9), 323-329 (2009).

(1)

E.C. Parent, S. Damaraju, D.L. Hill, E. Lou, and D. Smetaniuk, Identifying the best surface topography parameters for detecting idiopathic scoliosis curve progression, in Stud. Health Technol. Inform.(2010), pp. 78-82.

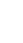

M. Mohokum, S. Mendoza, W. Udo, et al., Reproducibility of rasterstereography for kyphotic and lordotic angles, trunk length, and trunk inclination: a reliability study., Spine (Phila. Pa. 1976). 35(14), 1353-1358 (2010).

J. Schroeder, R. Reer, and K.M. Braumann, Video raster stereography back shape reconstruction: a reliability study for sagittal, frontal, and transversal plane parameters, Eur. Spine J. 24(2), 262-269 (2014).

T.C. Furian, W. Rapp, S. Eckert, M. Wild, and M. Betsch, Spinal posture and pelvic position in three hundred forty-five elementary school children: a rasterstereographic pilot study, Orthop. Rev. (Pavia). 5(1), 7 (2013).
A. Tabard-Fougère, A. Bonnefoy-Mazure, S. Hanquinet, P. Lascombes, S. Armand, and
R. Dayer, Validity and reliability of spine rasterstereography in patients with adolescent idiopathic scoliosis, Spine (Phila. Pa. 1976). 42(2), 98-105 (2017). 
P. Dankerl, A.K. Keller, L. Häberle, et al., Effects on posture by different neuromuscular afferent stimulations and proprioceptive insoles: Rasterstereographic evaluation, Prosthet. Orthot. Int. 40(3), 369-376 (2016). P. Knott, S. Mardjetko, D. Tager, R. Hund, and S. Thompson, The influence of body mass index (BMI) on the reproducibility of surface topography measurements, Scoliosis 7(Suppl 1), O18 (2012).

S. Goh, R.I. Price, P.J. Leedman, and K.P. Singer, Rasterstereographic Analysis of the Thoracic Sagittal Curvature: a Reliability Study, J. Musculoskelet. Res. 03(02), 137-142 (1999).

M. Betsch, M. Wild, P. Jungbluth, et al., Reliability and validity of 4D rasterstereography under dynamic conditions, Comput. Biol. Med. 41(6), 308-312 (2011).

P. Knott, S. Thompson, and S. Mardjetko, A Comparison of Automatic vs. Manual Detection of Anatomic Landmarks During Surface Topography Evaluation Using the Formetric 4D System, Scoliosis 7(Suppl 1), O19 (2012). A.F. Mannion, K. Knecht, G. Balaban, J. Dvorak, and D. Grob, A new skin-surface device for measuring the curvature and global and segmental ranges of motion of the spine: Reliability of measurements and comparison with data reviewed from the literature, Eur. Spine J. 13(2), 122-136 (2004). 
Table 1 Baseline demographic data for the whole sample and subdivided by gender.

\begin{tabular}{lccccc}
\hline & Count $\mathrm{n}(\%)$ & Age, years & Height, $\mathrm{m}$ & Weight, $\mathrm{kg}$ & $\mathrm{BMI}, \mathrm{kg} / \mathrm{m}^{2}$ \\
& & Mean (SD) & Mean (SD) & Mean (SD) & Mean (SD) \\
\hline All & $61(100 \%)$ & $37.5(12.4)$ & $1.7(0.1)$ & $72.3(19.9)$ & $26.0(6.1)$ \\
Female & $34(56 \%)$ & $37.1(12.6)$ & $1.6(0.1)$ & $68.0(15.6)$ & $25.3(5.2)$ \\
Male & $27(44 \%)$ & $38.8(12.5)$ & $1.8(0.1)$ & $76.3(22.0)$ & $26.9(7.1)$ \\
& & & & \\
& & & & & \\
\hline $\begin{array}{l}\text { SD, standard deviation. } \\
\text { BMl, body mass index. }\end{array}$ & & & & \\
\hline
\end{tabular}


Figure 1. 3D images of the spine and pelvis obtained using the Diers formetic 3D imaging system. The back surface reconstruction is illustrated with red areas highlighting the convex curvature and blue areas for the concave curvature. Yellow dots within each image demonstrate the axis for the coordinate system. The left and right lumbar dimples are at the bottom of the image (DL, DR) and the vertebra prominens (VP) of the top.

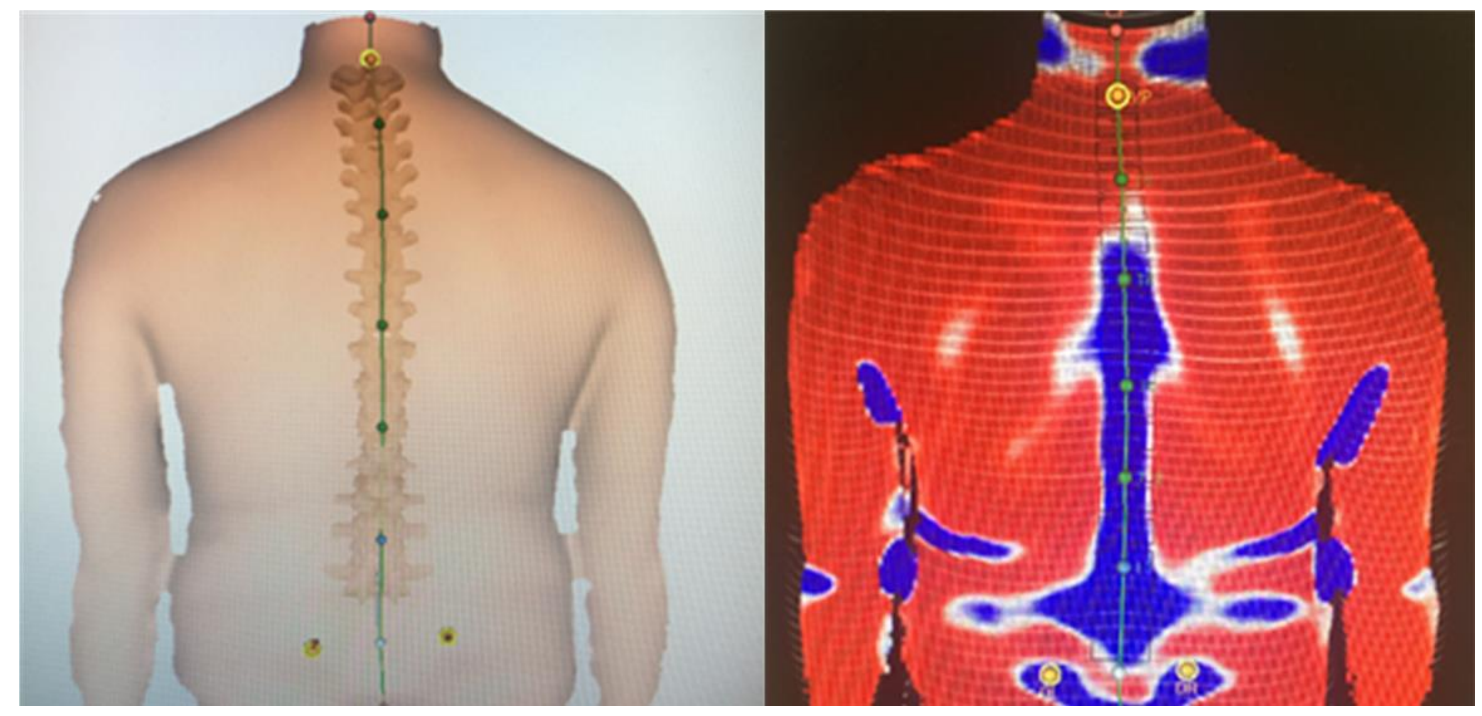


Figure 2. Illustrates the pelvic and spine measurements obtained during this study. $A: P T, B$ : PTor, $C: D D, D: T K,-\cdots$

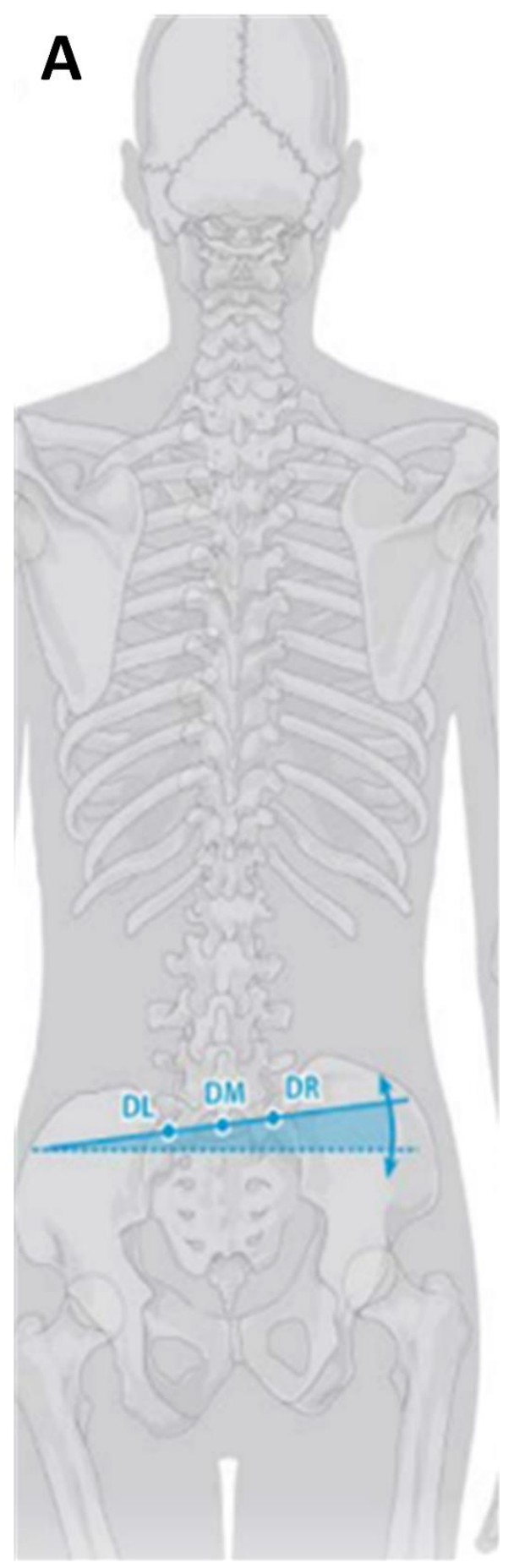



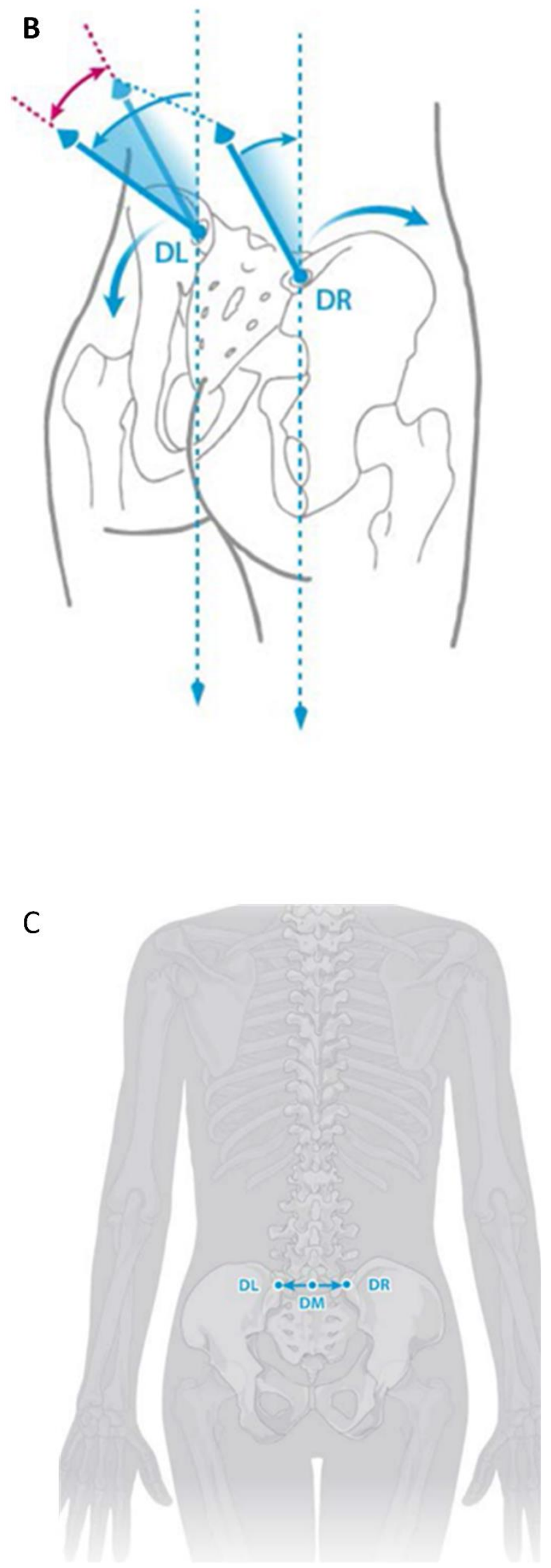


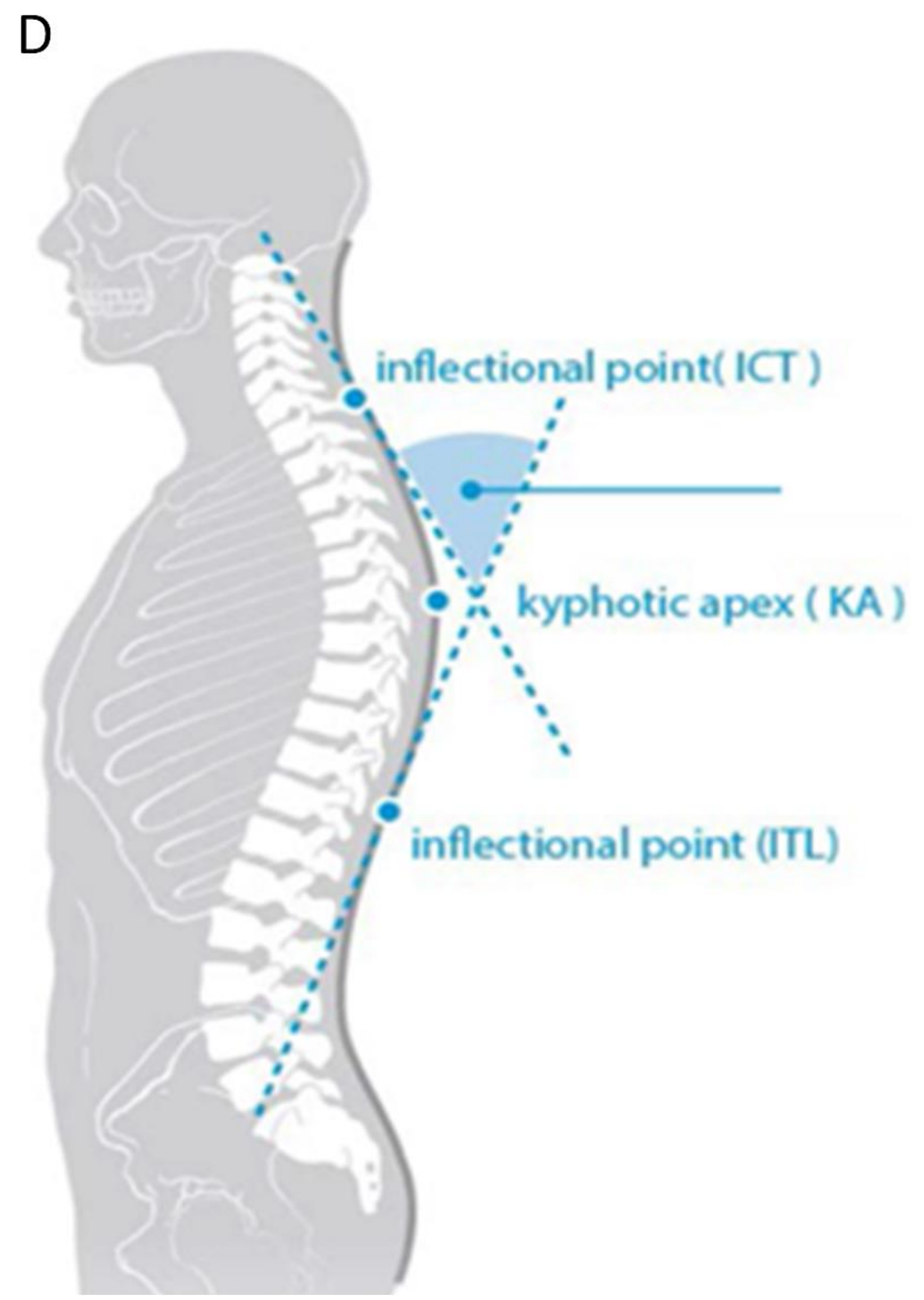


E

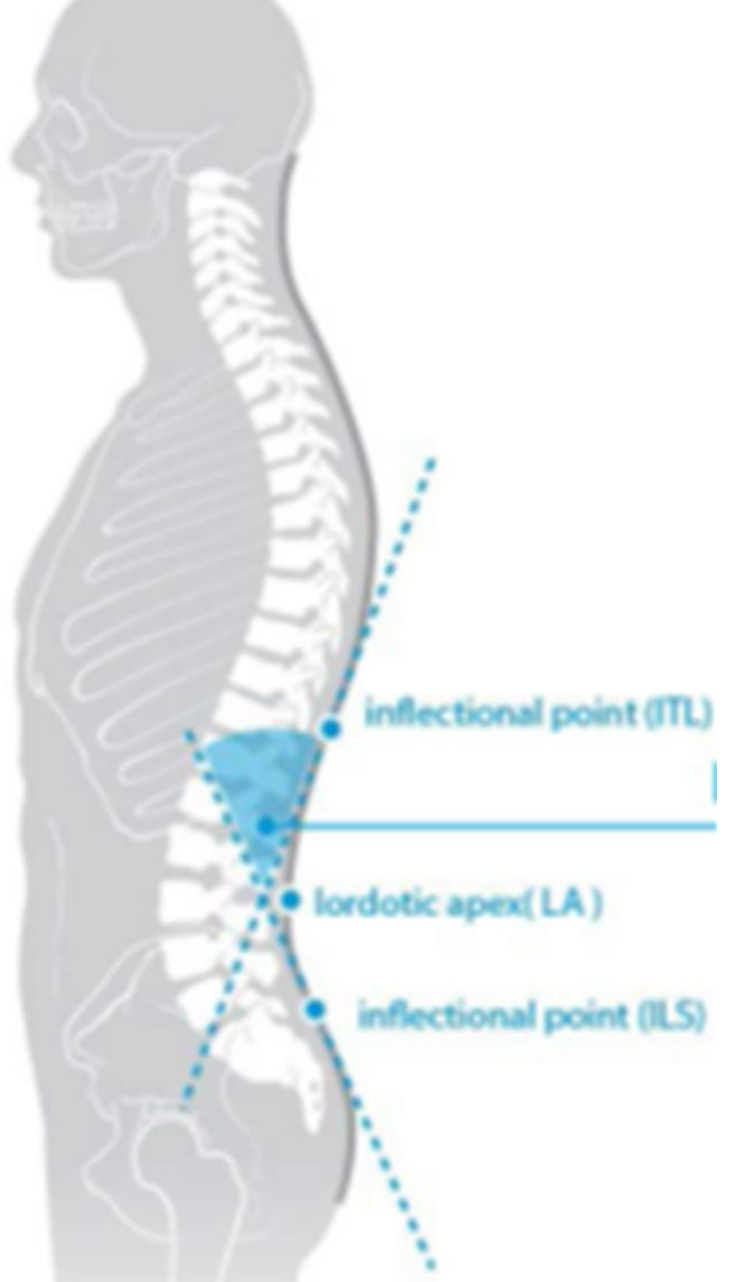


Table 2. Details of the eight different standing positions.

\begin{tabular}{|c|c|c|c|c|}
\hline Position & $\begin{array}{l}\text { Overall } \\
\text { Descript }\end{array}$ & & Feet position & Arms \\
\hline 1 & $\begin{array}{l}\text { Usual } \\
\text { position }\end{array}$ & standing & Neutral & By sides \\
\hline 2 & $\begin{array}{l}\text { Usual } \\
\text { position }\end{array}$ & standing & Neutral & Crossed over chest \\
\hline 3 & $\begin{array}{l}\text { Usual } \\
\text { position }\end{array}$ & standing & Neutral & $\begin{array}{l}\text { arms } 30^{\circ} \text { flexed fits touching the medial end } \\
\text { of the clavicle }\end{array}$ \\
\hline 4 & $\begin{array}{l}\text { Usual } \\
\text { position }\end{array}$ & standing & Neutral & arms $30^{\circ}$ flexed hands on support \\
\hline 5 & $\begin{array}{l}\text { Usual } \\
\text { position }\end{array}$ & standing & $\begin{array}{l}\text { Internally } \\
\text { rotated }\end{array}$ & By sides \\
\hline 6 & $\begin{array}{l}\text { Usual } \\
\text { position }\end{array}$ & standing & $\begin{array}{l}\text { Internally } \\
\text { rotated }\end{array}$ & Crossed over chest \\
\hline 7 & $\begin{array}{l}\text { Usual } \\
\text { position }\end{array}$ & standing & $\begin{array}{l}\text { Internally } \\
\text { rotated }\end{array}$ & $\begin{array}{l}\text { arms } 30^{\circ} \text { flexed fits touching the medial end } \\
\text { of the clavicle }\end{array}$ \\
\hline 8 & $\begin{array}{l}\text { Usual } \\
\text { position }\end{array}$ & standing & $\begin{array}{l}\text { Internally } \\
\text { rotated }\end{array}$ & arms $30^{\circ}$ flexed hands on support \\
\hline
\end{tabular}


Table 3. Summary statistics highlighting the variability in Diers measurements between the eight standing positions. The mean represents the average of three repeated measurements in one day.

\begin{tabular}{|c|c|c|c|c|c|c|c|c|c|c|c|c|c|c|c|}
\hline & \multicolumn{5}{|c|}{ MEAN $\pm S D / M E D I A N(I Q R)$} & \multicolumn{5}{|c|}{ SEm } & \multicolumn{5}{|c|}{$\mathrm{CV} \%$} \\
\hline $\begin{array}{l}\text { METRIC } \\
\text { /POSITI } \\
\text { ON }\end{array}$ & $\mathrm{DD}(\mathrm{mm})$ & PTor $\left({ }^{\circ}\right)$ & $\mathrm{PT}\left({ }^{\circ}\right)$ & $\mathrm{TK}\left({ }^{\circ}\right)$ & $\mathrm{LL}\left({ }^{\circ}\right)$ & $\mathrm{DD}(\mathrm{mm})$ & PTor $\left({ }^{\circ}\right)$ & $\mathrm{PT}\left({ }^{\circ}\right)$ & $\mathrm{TK}\left({ }^{\circ}\right)$ & $\mathrm{LL}\left({ }^{\circ}\right)$ & DD & PTor & PT & TK & LL \\
\hline 1 & $98.9(0.6)$ & $3.8(0.8)$ & $-1.1 \pm 0.8$ & $47.6 \pm 1.7$ & $36.2 \pm 1.6$ & 0.15 & 0.17 & 0.18 & 0.23 & 0.09 & $1 \%$ & $6 \%$ & $9 \%$ & $3 \%$ & $8 \%$ \\
\hline 2 & $99.0(0.4)$ & $3.5(0.1)$ & $-1.1 \pm 0.7$ & $47.5 \pm 1.6$ & $37.7 \pm 1.5$ & 0.01 & 0.28 & 0.15 & 0.20 & 0.09 & $0 \%$ & $6 \%$ & $1 \%$ & $2 \%$ & $6 \%$ \\
\hline 3 & $99.1(0.6)$ & $3.4(0.8)$ & $-1.1 \pm 0.8$ & $47.4 \pm 1.9$ & $37.9 \pm 1.6$ & 0.04 & 0.16 & 0.16 & 0.11 & 0.24 & $1 \%$ & $3 \%$ & $10 \%$ & $3 \%$ & $5 \%$ \\
\hline 4 & $99.4(0.4)$ & $3.4(1.0)$ & $-1.2 \pm 0.7$ & $48.4 \pm 1.7$ & $36.8 \pm 1.6$ & 0.02 & 0.30 & 0.15 & 0.25 & 0.22 & $0 \%$ & $5 \%$ & $9 \%$ & $3 \%$ & $5 \%$ \\
\hline 5 & $99.7(0.8)$ & $3.3(0.8)$ & $-0.9 \pm 1.0$ & $48.0 \pm 1.8$ & $37.3 \pm 1.3$ & 0.13 & 0.18 & 0.24 & 0.28 & 0.16 & $0 \%$ & $1 \%$ & $7 \%$ & $3 \%$ & $4 \%$ \\
\hline 7 & $99.7(0.7)$ & $3.4(0.8)$ & $-0.8 \pm 0.5$ & $47.0 \pm 1.8$ & $39.1 \pm 1.3$ & 0.04 & 0.17 & 0.09 & 0.31 & 0.15 & $0 \%$ & $1 \%$ & $7 \%$ & $4 \%$ & $2 \%$ \\
\hline 8 & $99.2(0.4)$ & $3.6(0.8)$ & $-0.9 \pm 0.4$ & $48.0 \pm 1.8$ & $38.0 \pm 1.4$ & 0.00 & 0.16 & 0.05 & 0.25 & 0.15 & $0 \%$ & $3 \%$ & $10 \%$ & $3 \%$ & $3 \%$ \\
\hline
\end{tabular}

DD: Dimple Distance; PTor: Pelvis Torsion; PT: pelvis tilt; TK: Thoracic Kyphosis; LL: Lumber lordosis.

CV\%: Coefficient of variation; SEm: standard error of measurements. 
Table 4 Statistical analysis of group differences between the three repeated measures

\begin{tabular}{|c|c|c|c|c|c|c|c|c|c|c|c|c|c|c|c|}
\hline \multicolumn{16}{|c|}{ Group mean differences } \\
\hline \multirow[t]{2}{*}{$\begin{array}{l}\text { METRIC } \\
\text { /POSITION }\end{array}$} & \multicolumn{3}{|c|}{$\mathrm{DD}(\mathrm{mm})^{1}$} & \multicolumn{3}{|c|}{ PTor $\left({ }^{\circ}\right)^{2}$} & \multicolumn{3}{|c|}{ PT $\left({ }^{\circ}\right)^{3}$} & \multicolumn{3}{|c|}{ TK $\left({ }^{\circ}\right)^{4}$} & \multicolumn{3}{|c|}{$\mathrm{LL}\left({ }^{\circ}\right)^{5}$} \\
\hline & $\mathrm{F}$ & $P$ & $\eta 2$ & $\mathrm{~F}$ & $P$ & $\eta 2$ & $\mathrm{~F}$ & $P$ & $\eta 2$ & $\mathrm{~F}$ & $P$ & $\eta 2$ & $\mathrm{~F}$ & $P$ & $\eta 2$ \\
\hline 1 & 5.6 & 0.05 & 0.166 & 0.185 & 0.832 & 0.006 & 0.164 & 0.849 & 0.006 & 0.82 & 0.44 & 0.029 & 1.66 & 0.199 & 0.055 \\
\hline 2 & 3.4 & 0.07 & 0.109 & 2.02 & 0.141 & 0.066 & 1.57 & 0.216 & 0.052 & 1.02 & 0.36 & 0.035 & 0.645 & 0.528 & 0.002 \\
\hline 3 & 0.22 & 0.79 & 0.008 & 0.858 & 0.429 & 0.029 & 0.199 & 0.820 & 0.007 & 0.87 & 0.42 & 0.03 & 1.18 & 0.313 & 0.040 \\
\hline 4 & 0.94 & 0.17 & 0.059 & 1.77 & 0.179 & 0.059 & 0.203 & 0.817 & 0.007 & 1.42 & 0.25 & 0.04 & 1.89 & 0.159 & 0.062 \\
\hline 5 & 0.42 & 0.65 & 0.015 & 1.05 & 0.356 & 0.036 & 1.29 & 0.281 & 0.044 & 2.31 & 0.11 & 0.076 & 0.72 & 0.487 & 0.025 \\
\hline 6 & 0.49 & 0.61 & 0.01 & 1.28 & 0.286 & 0.043 & 0.597 & 0.554 & 0.021 & 0.340 & 0.714 & 0.012 & 2.22 & 0.117 & 0.073 \\
\hline 7 & 1.31 & 0.27 & 0.044 & 0.225 & 0.779 & 0.008 & 0.827 & 0.442 & 0.028 & 0.30 & 0.740 & 0.011 & 1.42 & 0.249 & 0.048 \\
\hline 8 & 0.13 & 0.87 & 0.005 & 0.160 & 0.853 & 0.006 & 0.253 & 0.778 & 0.009 & 2.42 & 0.098 & 0.080 & 1.35 & 0.266 & 0.045 \\
\hline
\end{tabular}


Table 5. Reliability coefficients (ICC $\pm \mathrm{Cl} 95 \%$ ) of pelvis and spine in three repeated measurements and among all positions

\begin{tabular}{|c|c|c|c|c|c|}
\hline \multirow[b]{2}{*}{$\begin{array}{l}\text { METRIC } \\
\text { /POSITION }\end{array}$} & \multicolumn{4}{|c|}{ ICC $( \pm 95 \% \mathrm{Cl})$} & \multirow[b]{2}{*}{$\mathrm{LL}$} \\
\hline & DD & PTor & PT & $\mathrm{TK}$ & \\
\hline 1 & $0.999(0.998-0.999)^{*}$ & $0.956(0.932-0.972)^{*}$ & $0.952(0.926-0.970)^{*}$ & $0.982(0.972-0.989)^{*}$ & $0.997(0.964-0.985)^{*}$ \\
\hline 2 & $0.999(0.999-1)^{*}$ & $0.879(0.813-0.924)^{*}$ & $0.964(0.944-0.977)^{*}$ & $0.986(0.978-0.991)^{*}$ & $0.997(0.965-0.986)^{*}$ \\
\hline 3 & $0.996(0.993-0.997)^{*}$ & $0.961(0.940-0.975)^{*}$ & $0.962(0.942-0.976)^{*}$ & $0.997(0.965-0.986)^{*}$ & $0.976(0.964-0.985)^{*}$ \\
\hline 4 & $0.999(0.999-0.999)^{*}$ & $0.941(0.910-0.963)^{*}$ & $0.956(0.932-0.972)^{*}$ & $0.979(0.968-0.987)^{*}$ & $0.980(0.969-0.987)^{*}$ \\
\hline 5 & $0.972(0.956-0.982)^{*}$ & $0.959(0.936-0.974)^{*}$ & $0.936(0.902-0.960)^{*}$ & $0.975(0.961-0.984)^{*}$ & $0.984(0.975-0.990)^{*}$ \\
\hline 6 & $1(0.999-1)^{*}$ & $0.963(0.943-0.977)^{*}$ & $0.970(0.954-0.981)^{*}$ & $0.985(0.976-0.990)^{*}$ & $0.989(0.982-0.993)^{*}$ \\
\hline 7 & $0.997(0.964-0.986)^{*}$ & $0.962(0.941-0.976)^{*}$ & $0.976(0.963-0.963)^{*}$ & $0.969(0.952-0.980)^{*}$ & $0.986(0.978-0.991)^{*}$ \\
\hline 8 & $1(0.999-1)^{*}$ & $0.962(0.941-0.976)^{*}$ & $0.986(0.979-0.992)^{*}$ & $0.979(0.967-0.987)^{*}$ & $0.987 .980-0.992)^{*}$ \\
\hline
\end{tabular}

DD: Dimple Distance; PTor: Pelvis Torsion; PT: pelvis tilt; TK: Thoracic Kyphosis; LL: Lumber lordosis Level of significant $\mathrm{P}<0.001$ 
